\title{
Probability-Dependent Static Output Feedback Control for Discrete-Time Nonlinear Stochastic Systems with Missing Measurements
}

\author{
Wangyan Li, Guoliang Wei, and Licheng Wang \\ Department of Control Science and Engineering, University of Shanghai for Science and Technology, \\ Shanghai 200093, China \\ Correspondence should be addressed to Guoliang Wei, guoliang.wei1973@gmail.com
}

Received 21 June 2012; Accepted 2 August 2012

Academic Editor: Zidong Wang

Copyright (C) 2012 Wangyan Li et al. This is an open access article distributed under the Creative Commons Attribution License, which permits unrestricted use, distribution, and reproduction in any medium, provided the original work is properly cited.

\begin{abstract}
This paper is devoted to the problems of gain-scheduled control for a class of discretetime stochastic systems with infinite-distributed delays and missing measurements by utilizing probability-dependent Lyapunov functional. The missing-measurement phenomenon is assumed to occur in a random way, and the missing probability is time varying with securable upper and lower bounds that can be measured in real time. The purpose is to design a static output feedback controller with scheduled gains such that, for the admissible random missing measurements, time delays, and noises, the closed-loop system is exponentially mean-square stable. At last, a simulation example is exploited to illustrate the effectiveness of the proposed design procedures.
\end{abstract}

\section{Introduction}

Gain-scheduling is one of the most popular methods of controller design and has been extensively applied in engineering, such as rotation speed control of engine, aircraft control and process control. Over the past decades, the gain-scheduled control problem has been extensively studied both from theoretical and practical viewpoint, see, for example, [1-6]. For the controller design problems for parameter-varying systems, the gain-scheduling approach has been found to be one of the most effective ones, whose main idea is to design controller gains as functions of the scheduling parameters, which are supposed to be available in real time and, therefore, have much less conservatism than the conventional ones.

On the other hand, instead of using the information of system states, static output feedback (SOF) control directly makes use of system outputs to design controllers, which has also attracted attentions of many researchers over the past two decades, see, for 
example, [7-12]. It is obvious that the structure of SOF controllers is simple and easy to implement. However, to the best of the authors' knowledge, there has been little research attention on the control problem for discrete-time nonlinear stochastic systems with a missing phenomenon based on the time-varying occurring probability by a gain-scheduling method.

The missing-measurement phenomenon, due to various reasons such as probabilistic network congestion and intermittent mechanical failures, usually occurs in many real-world systems, which has attracted considerable attention during the past few years, see, for example, [13-15]. The Bernoulli distribution has been successfully applied to model this phenomenon, in which 0 is used to stand for an entire signal missing and 1 denotes the intactness (i.e., there is no signal missing at all), and all sensors have the same missing probability, which is simple and effective and has become very popular during the past years, see, for example, $[5,13,14,16]$. However, in the practical systems, the occurring probability of the missing-measurement phenomenon might be time varying; consequently, a time-varying Bernoulli distribution model is more suitable for such parameter-varying systems.

In another aspect, considering the signal propagation often distributed during a certain time period, then, a new kind of delays, namely, distributed time-delays, has drawn many researchers' attention, see, for example, [17-22], but most of the existing works on distributed delays have focused on continuous-time systems which are described either in the form of finite or infinite integral. As we all know, when it comes to implementing the control laws in a digital way, the discrete-time system is much better than continuous-time one. Naturally, it turns out to be meaningful to investigate the issue of how distributed delays influence the dynamical behavior of a discrete-time system. However, as far as authors know, based on gain-scheduled control methods, the SOF control problem for nonlinear stochastic systems with infinite-distributed delays and missing measurements with time-varying occurring probability has not been addressed yet and is still a very interesting and challenging problem.

The main contributions of this paper are summarized as follows: (1) a new SOF control problem is addressed for a class of discrete-time nonlinear stochastic systems with missing measurements and infinite-distributed delays via a gain-scheduling approach; (2) a sequence of stochastic variables satisfying Bernoulli distributions is introduced to describe the timevarying features of the missing measurements in the sensor; (3) a time-varying Lyapunov functional dependent on the missing probability is proposed and then applied to improve the performance of the gain-scheduled controller; and (4) a gain-scheduled controller is designed, in which the controller parameters can be adjusted online according to the missing probabilities estimated through statistical tests.

Notation 1 . In this paper, $\mathbb{R}^{n}, \mathbb{R}^{n \times m}$, and $\mathbb{I}^{+}$denote, respectively, the $n$-dimensional Euclidean space, and the set of all $n \times m$ real matrices, the set of all positive integers. $|\cdot|$ refers to the Euclidean norm in $\mathbb{R}^{n}$. I denotes the identity matrix of compatible dimension. The notation $X \geq Y$ (resp., $X>Y$ ), where $X$ and $Y$ are symmetric matrices, means that $X-Y$ is positive semidefinite (resp., positive definite). For a matrix $M, M^{T}$ and $M^{-1}$ represent its transpose and inverse, respectively. The shorthand $\operatorname{diag}\left\{M_{1}, M_{2}, \ldots, M_{n}\right\}$ denotes a block diagonal matrix with diagonal blocks being the matrices $M_{1}, M_{2}, \ldots, M_{n}$. In symmetric block matrices, the symbol $*$ is used as an ellipsis for terms induced by symmetry. Matrices, if they are not explicitly stated, are assumed to have compatible dimensions. In addition, $\mathbb{E}\{x\}$ and $\operatorname{Prob}\{y\}$ will, respectively, mean expectation of $x$ and probability of $y$. 


\section{Problem Formulation}

Consider the following discrete-time nonlinear stochastic systems with infinite-distributed delays:

$$
\begin{gathered}
x(k+1)=A x(k)+B u(k)+D \sum_{d=1}^{+\infty} \mu_{d} x(k-d)+N f(z(k))+E x(k) w(k), \\
x(k)=\rho(k), \quad k=-d,-d+1, \ldots, 0,
\end{gathered}
$$

where $x(k) \in \mathbb{R}^{n}$ is the state, $z(k):=G x(k)+G_{d} \sum_{d=1}^{+\infty} \mu_{d} x(k-d) . \omega(k)$ is a one-dimensional Gaussian white noise sequence satisfying $\mathbb{E}\{\omega(k)\}=0$ and $\mathbb{E}\left\{\omega^{2}(k)\right\}=\sigma^{2}, \rho(k)$ is the initial state of the system. $A, B, D, N, E, G$, and $G_{d}$ are constant real matrices of appropriate dimensions and $B$ is of full-column rank.

The nonlinear function $f(\cdot)$ with $(f(0)=0)$ is assumed as nonlinear disturbances and satisfies the following sector-bounded condition:

$$
\left[f(z(k))-F_{1} z(k)\right]^{T}\left[f(z(k))-F_{2} z(k)\right] \leq 0,
$$

where $f(\cdot)$ is called to belong to the sector $\left[F_{1}, F_{2}\right]$ and $F_{1}$ and $F_{2}$ are given constant real matrices.

For the technique convenience, the nonlinear function $f(z(k))$ can be decomposed into a linear and a nonlinear part as

$$
f(z(k))=f_{s}(z(k))+F_{1} z(k)
$$

then, from (2.3), we have

$$
f_{s}^{T}(z(k))\left(f_{s}(z(k))-F z(k)\right) \leq 0,
$$

where $F=F_{2}-F_{1}>0$.

On the other hand, $\mu_{d} \geq 0$ is the convergence constant that satisfies the following condition:

$$
\sum_{d=1}^{+\infty} \mu_{d} \leq \sum_{d=1}^{+\infty} d \mu_{d}<+\infty
$$

Remark 2.1. The distributed delay is one important type of time delays and has been widely recognized and intensively studied, see, for example, [17-22]. The delay term $\sum_{d=1}^{+\infty} \mu_{d} x(k-d)$ in the resulted stochastic system (2.1) called infinitely distributed delay. However, almost all existing references concerning distributed delays are concerned with the continuous-time systems, where the distributed delays are described in the form of a finite or infinite integral. In this paper, the constants $\mu_{d}(d=1,2, \ldots)$ are assumed to satisfy the convergence conditions (2.6), which can guarantee the convergence of the terms of infinite delays as well as the Lyapunov-Krasovskii functional defined later. 
The measurement output with missing sensor data is described as

$$
y(k)=\xi(k) C x(k),
$$

where $C$ is a constant real matrix of appropriate dimensions and $\xi(k) \in \mathbb{R}$ is a random white sequence characterizing the probabilistic sensor-data missing, which obeys the following time-varying Bernoulli distribution:

$$
\begin{gathered}
\operatorname{Prob}\{\xi(k)=1\}=\mathbb{E}\{\xi(k)\}=p(k), \\
\operatorname{Prob}\{\xi(k)=0\}=1-\mathbb{E}\{\xi(k)\}=1-p(k),
\end{gathered}
$$

where $p(k)$ is a time-varying positive scalar sequence and belongs to $\left[p_{1} p_{2}\right] \subseteq[01]$ with $p_{1}$ and $p_{2}$ being the lower and upper bounds of $p(k)$, respectively. In this paper, for simplicity, we assume that $\xi(k), \omega(k)$ and $\rho(k)$ are uncorrelated.

Remark 2.2. In (2.7), a random white sequence satisfying the time-varying Bernoulli distribution is introduced to reflect the missing-measurement phenomenon that has attracted considerable attention in the past few years, see, for example, [13-15]. However, the missing probability in most relevant literatures has always been assumed to be a constant. Such an assumption, unfortunately, tends to be conservative in handling time-varying missing measurements. In this paper, the missing probability is allowed to be time-varying with known lower and upper bounds, which will then be used to schedule controller gains, thereby reducing the possible conservatism.

In this paper, we are interested in designing the following gain-scheduled controller:

$$
u(k)=K(p) y(k),
$$

where $K(p)$ is the controller gain sequence to be designed and assumed as the following structure:

$$
K(p)=K_{0}+p(k) K_{u}
$$

for every time step $k, p(k)$ is the time-varying parameter of the controller gain, which takes value in $\left[p_{1}, p_{2}\right]$ and $K_{0}, K_{u}$ are the constant parameters of the controller gain to be designed. The closed-loop system of the static output feedback gain-scheduled controller is as follows:

$$
x(k+1)=A x(k)+\xi(k) B K(p) C x(k)+D \sum_{d=1}^{+\infty} \mu_{d} x(k-d)+N f(z(k))+E x(k) w(k) .
$$

Before formulating the problem to be investigated, we first introduce the following stability concepts. 
Definition 2.3. The closed-loop system (2.11) is said to be exponentially mean-square stable if, with $w(k)=0$, there exist constants $\alpha>0$ and $\tau \in(0,1)$ such that

$$
\mathbb{E}\left\{\|\eta(k)\|^{2}\right\} \leq \alpha \tau^{k} \sup _{-d \leq i \leq 0} \mathbb{E}\left\{\|\eta(i)\|^{2}\right\}, \quad k \in \mathbb{I}^{+}
$$

In this paper, our purpose is to design a probability-dependent gain-scheduled controller of the form (2.9) for the system (2.1) by exploiting a probability-dependent Lyapunov functional and LMI method such that, for all admissible infinite-distributed delays, missing measurements with time-varying probability, and exogenous stochastic noises, the closed-loop system (2.11) is exponentially mean-square stable.

\section{Main Results}

The following lemmas will be used in the proofs of our main results in this paper.

Lemma 3.1 ([Schur complement] see[23]). Given constant matrices $\Sigma_{1}, \Sigma_{2}, \Sigma_{3}$ where $\Sigma_{1}=\Sigma_{1}^{T}$ and $0<\Sigma_{2}=\Sigma_{2}^{T}$, then $\Sigma_{1}+\Sigma_{3}^{T} \Sigma_{2}^{-1} \Sigma_{3} \geq 0$ if and only if

$$
\left[\begin{array}{cc}
\Sigma_{1} & \Sigma_{3}^{T} \\
\Sigma_{3} & -\Sigma_{2}
\end{array}\right] \geq 0 \text { or } \quad\left[\begin{array}{cc}
-\Sigma_{2} & \Sigma_{3} \\
\Sigma_{3}^{T} & \Sigma_{1}
\end{array}\right] \geq 0
$$

Lemma 3.2 (see [24]). Let $M \in \mathbb{R}^{n \times n}$ be a positive semidefinite matrix, $x_{i} \in \mathbb{R}^{n}$ and constant $a_{i}>$ $0(i=1,2, \ldots)$. If the series concerned is convergent, then one has

$$
\left(\sum_{i=1}^{\infty} a_{i} x_{i}\right)^{T} M\left(\sum_{i=1}^{\infty} a_{i} x_{i}\right) \leq\left(\sum_{i=1}^{\infty} a_{i}\right) \sum_{i=1}^{\infty} a_{i} x_{i}^{T} M x_{i} .
$$

Lemma 3.3 (see [25]). Let the matrix $B \in R^{n \times m}$ be of full-column rank. There always exist two orthogonal matrices $U \in R^{n \times n}$ and $V \in R^{n \times n}$ such that

$$
\begin{gathered}
B=U\left[\begin{array}{l}
\Sigma \\
0
\end{array}\right] V^{T}, \\
\Sigma=\operatorname{diag}\left\{\sigma_{1}, \sigma_{2}, \ldots, \sigma_{m}\right\} .
\end{gathered}
$$

If matrix $S$ has the following structure:

$$
S=U\left[\begin{array}{cc}
S_{11} & S_{12} \\
0 & S_{22}
\end{array}\right] U^{T}
$$

where $S_{11} \in R^{n \times m}, S_{12} \in R^{n \times(n-m)}, S_{22} \in R^{(n-m) \times(n-m)}$, then there exists a nonsingular matrix $R \in R^{m \times m}$ such that $S B=B R$.

In the following theorem, a probability-dependent gain-scheduled static output feedback control problem is dealt with for a class of discrete-time nonlinear stochastic systems 
(2.1) by exploiting Lyapunov theory and LMI method. A sufficient condition is derived to guarantee the solvability of the desired gain-scheduled control problem and, simultaneously, the parameters of the gain-scheduled controller can be obtained by solving the LMIs and the measured time-varying probability.

Theorem 3.4. Consider the discrete-time nonlinear stochastic systems (2.11). If there exist positivedefinite matrices $Q(p(k))$ and $Q_{\tau}$, slack matrix $S$ and nonsingular matrices $Y(p)$ and $R$, such that the following LMIs hold:

$$
\left[\begin{array}{cccccc}
\bar{\mu} Q_{\tau}-Q(p(k)) & * & * & * & * & * \\
0 & -\frac{1}{\bar{\mu}} Q_{\tau} & * & * & * & * \\
F G & F G_{d} & -2 I & * & * & * \\
S^{T} \bar{A}+p(k) B Y(p) C & S^{T} \bar{D} & S^{T} N & -\bar{\Lambda} & * & * \\
\sigma^{2} S^{T} E & 0 & 0 & 0 & -\sigma^{2} \bar{\Lambda} & * \\
\Delta_{p}(k) B Y(p) C & 0 & 0 & 0 & 0 & -\Delta_{p}(k) \bar{\Lambda}
\end{array}\right]<0
$$

where

$$
\begin{gathered}
\bar{\Lambda}=-Q(p(k+1))+S+S^{T}, \quad \bar{\mu}=\sum_{d=1}^{+\infty} \mu_{d}, \quad \Delta_{P}(k)=P(k)(1-P(k)), \\
\bar{A}=A+N F_{1} G, \quad \bar{D}=D+N F_{1} G_{d}, \\
S^{T} B=B R, \quad R K(p)=Y(p), \quad K(p)=R^{-1} Y(p),
\end{gathered}
$$

in this case, the constant gains of the desired controller can be obtained as follows:

$$
K_{0}=R^{-1} Y_{0}, \quad K_{u}=R^{-1} Y_{u}
$$

and the closed-system (2.11) is then exponentially mean-square stable for all $p(k) \in\left[\begin{array}{ll}p_{1} & p_{2}\end{array}\right]$.

Proof. Define the Lyapunov functional:

$$
V(k):=x^{T}(k) Q(p(k)) x(k)+\sum_{d=1}^{+\infty} \mu_{d} \sum_{s=k-d}^{k-1} x^{T}(s) Q_{\tau} x(s) .
$$


Then, noting $\mathbb{E}\{\xi(k)-p(k)\}=0, \mathbb{E}\{\omega(k)\}=0$ and $\mathbb{E}\left\{[\xi(k)-p(k)]^{2}\right\}=p(k)(1-p(k))$, we can get that

$$
\begin{aligned}
& \mathbb{E}\{\Delta V(k)\}=\mathbb{E}\left\{x^{T}(k+1) Q(p(k+1)) x(k+1)-x^{T}(k) Q(p(k)) x(k)+\bar{\mu} x^{T}(k) Q_{\tau} x(k)\right. \\
&\left.\quad-\sum_{d=1}^{+\infty} \mu_{d} x^{T}(k-d) Q_{\tau} x(k-d)\right\} \\
& \leq \mathrm{E}\left\{\left[(\bar{A}+p(k) B K(p) C) x(k)+\bar{D} \sum_{m=1}^{+\infty} \mu_{d} x(k-d)+N f_{s}(z(k))\right]^{T} Q(p(k+1))\right. \\
& \times\left[(\bar{A}+p(k) B K(p) C) x(k)+\bar{D} \sum_{m=1}^{+\infty} \mu_{d} x(k-d)+N f_{s}(z(k))\right] \\
&+[p(k)(1-(p(k)) B K(p) C) x(k)]^{T} Q(p(k+1)) B K(p) C x(k)+\sigma^{2} x^{T}(k) E^{T} \\
& \times Q(p(k+1)) E x(k)-x^{T}(k) Q(p(k)) x(k)-\sum_{d=1}^{+\infty} \mu_{d} x^{T}(k-d) Q_{\tau} x(k-d) \\
&+\bar{\mu} x^{T}(k) Q_{\tau} x(k)+2 f_{s}^{T}(z(k)) F G x(k)+2 f_{s}^{T}(z(k)) F G_{d} \sum_{m=1}^{+\infty} \mu_{d} x(k-d) \\
&\left.-2 f_{s}^{T}(z(\mathrm{k})) f_{s}(z(k))\right\} .
\end{aligned}
$$

From Lemma 3.2, it is obvious that

$$
-\sum_{d=1}^{+\infty} \mu_{d}\left(x^{T}(k-d) Q_{\tau} x(k-d)\right) \leq-\frac{1}{\bar{\mu}}\left(\sum_{d=1}^{+\infty} \mu_{d} x^{T}(k-d)\right) Q_{\tau}\left(\sum_{d=1}^{+\infty} \mu_{d} x(k-d)\right)
$$

Denote the following matrix variables

$$
\eta(k)=\left[\begin{array}{lll}
x^{T}(k) & \sum_{d=1}^{+\infty} \mu_{d} x^{T}(k-d) & f_{s}^{T}(z(k))
\end{array}\right]^{T} .
$$


Combining (3.9), (3.10), and (3.11), we can get

$$
\begin{gathered}
\mathbb{E}\{\Delta V(k)\} \leq \mathbb{E}\left\{\eta^{T}(k) \Omega \eta(k)\right\}, \\
\Omega=\left[\begin{array}{ccc}
\Omega_{1} & * & * \\
\Omega_{2} & \Omega_{3} & * \\
\Omega_{4} & \Omega_{5} & \Omega_{6}
\end{array}\right], \\
\Omega_{1}=(\bar{A}+p(k) B K(p) C)^{T} Q(p(k+1))(\bar{A}+p(k) B K(p) C)+\sigma^{2} E^{T} Q(p(k+1)) E \\
+p(k)(1-p(k))(B K(p) C)^{T} Q(p(k+1)) B K(p) C+\bar{\mu} Q_{\tau}-Q(p(k)), \\
\Omega_{2}=\bar{D}^{T} Q(p(k+1))(\bar{A}+p(k) B K(p) C), \\
\Omega_{3}=\bar{D}^{T} Q(p(k+1)) \bar{D}-\frac{1}{\bar{\mu}} Q_{\tau}, \\
\Omega_{4}=N^{T} Q(p(k+1))(\bar{A}+p(k) B K(p) C)+F G, \\
\Omega_{5}=N^{T} Q(p(k+1)) \bar{D}+F G_{d}, \\
\Omega_{6}=N^{T} Q(p(k+1)) N-2 I .
\end{gathered}
$$

If $\Omega \leq 0$, we can conclude the following matrix inequalities by Schur complement:

$$
\left[\begin{array}{cccccc}
\bar{\mu} Q_{\tau}-Q(p(k)) & * & * & * & * & * \\
0 & -\frac{1}{\bar{\mu}} Q_{\tau} & * & * & * & * \\
F G & F G_{d} & -2 I & * & * & * \\
\bar{A}+p(k) B K(p) C & \bar{D} & N & -\Lambda & * & * \\
E & 0 & 0 & 0 & -\sigma^{-2} \Lambda & * \\
B K(p) C & 0 & 0 & 0 & 0 & -\Delta_{p}^{-1}(k) \Lambda
\end{array}\right]<0
$$

with $\Lambda=Q^{-1}(p(k+1))$.

At this time, preforming the congruence transformation $\operatorname{diag}\left\{I, I, I, S, \sigma^{2} S, \Delta_{p}(k) S\right\}$ to (3.13), we can have

$$
\begin{gathered}
{\left[\begin{array}{cccccc}
\bar{\mu} Q_{\tau}-Q(p(k)) & * & * & * & * & * \\
0 & -\frac{1}{\bar{\mu}} Q_{\tau} & * & * & * & * \\
F G & F G_{d} & -2 I & * & * & * \\
S^{T} \bar{A}+p(k) S^{T} B K(p) C & S^{T} \bar{D} & S^{T} N & -\widehat{\Lambda} & * & * \\
\sigma^{2} S^{T} E & 0 & 0 & 0 & -\sigma^{2} \widehat{\Lambda} & * \\
\Delta_{p}(k) S^{T} B K(p) C & 0 & 0 & 0 & 0 & -\Delta_{p}(k) \widehat{\Lambda}
\end{array}\right]<0,} \\
\widehat{\Lambda}=S^{T} Q^{-1}(p(k+1)) S,
\end{gathered}
$$


then from inequality

$$
S^{T} Q^{-1}(p(k+1)) S \geq S^{T}+S-\mathrm{Q}(p(k+1))=\bar{\Lambda},
$$

we can get

$$
\left[\begin{array}{cccccc}
\bar{\mu} Q_{\tau}-Q(p(k)) & * & * & * & * & * \\
0 & -\frac{1}{\bar{\mu}} Q_{\tau} & * & * & * & * \\
F G & F G_{d} & -2 I & * & * & * \\
S^{T} \bar{A}+p(k) S^{T} B K(p) C & S^{T} \bar{D} & S^{T} N & -\bar{\Lambda} & * & * \\
\sigma^{2} S^{T} E & 0 & 0 & 0 & -\sigma^{2} \bar{\Lambda} & * \\
\Delta_{p}(k) S^{T} B K(p) C & 0 & 0 & 0 & 0 & -\Delta_{p}(k) \bar{\Lambda}
\end{array}\right]<0
$$

and from lemma 3, we have $S^{T} B=B R$ denoting $R K(p)=Y(p)$, and $K(P)=R^{-1} Y(p)$. Then (3.16) can be written as

$$
\left[\begin{array}{cccccc}
\bar{\mu} Q_{\tau}-Q(p(k)) & * & * & * & * & * \\
0 & -\frac{1}{\bar{\mu}} Q_{\tau} & * & * & * & * \\
F G & F G_{d} & -2 I & * & * & * \\
S^{T} \bar{A}+p(k) B Y(p) C & S^{T} \bar{D} & S^{T} N & -\bar{\Lambda} & * & * \\
\sigma^{2} S^{T} E & 0 & 0 & 0 & -\sigma^{2} \bar{\Lambda} & * \\
\Delta_{p}(k) B Y(p) C & 0 & 0 & 0 & 0 & -\Delta_{p}(k) \bar{\Lambda}
\end{array}\right]<0
$$

Furthermore, by Lemma 3.1, we can know from that $\Omega<0$ and, subsequently,

$$
\mathbb{E}\{\Delta V(k)\}<-\lambda_{\min }(-\Omega) \mathbb{E}|\eta(k)|^{2},
$$

where $\lambda_{\min }(-\Omega)$ is the minimum eigenvalue of $(-\Omega)$. Finally, we can confirm from Lemma 1 of [13] that the closed-loop system is exponentially mean-square stable, then the proof of this theorem is complete.

Remark 3.5. In the above theorem, a static output feedback controller has been designed based on a set of LMIs. However, the LMIs are actually infinite owing to the time-varying parameter $p(k) \in\left[p_{1} p_{2}\right]$. In this case, the desired controller cannot be obtained directly from Theorem 3.4 due to the infinite number of LMIs. To handle such a problem, in the next theorem, we have to convert this problem to a computationally accessible one by assigning a specific form to $p(k)$. Let us set $Q(p(k))=Q_{0}+p(k) Q_{u}$. 
Theorem 3.6. Consider the discrete-time nonlinear stochastic system with infinite-distributed delays and missing measurements (2.11). If there exist positive-difinite matrices $Q_{0}, Q_{u}$ and $Q_{\tau}$, slack matrix $S$ and nonsingular matrices $Y(p)$ and $R$, such that the following LMIs hold:

$$
M^{i j l m}=\left[\begin{array}{cccccc}
\bar{\mu} Q_{\tau}-Q^{i}(p(k)) & * & * & * & * & * \\
0 & -\frac{1}{\bar{\mu}} Q_{\tau} & * & * & * & * \\
F G & F G_{d} & -2 I & * & * & * \\
S^{T} \bar{A}+p_{i} B Y^{m} C & S^{T} \bar{D} & S^{T} N & -\bar{\Lambda}^{l} & * & * \\
\sigma^{2} S^{T} E & 0 & 0 & 0 & -\sigma^{2} \bar{\Lambda}^{l} & * \\
\Delta^{i j} B Y^{m} C & 0 & 0 & 0 & 0 & -\Delta^{i j} \bar{\Lambda}^{l}
\end{array}\right]<0,
$$

where

$$
\begin{gathered}
\bar{\Lambda}^{l}=-Q_{0}-p_{l} Q_{u}+S+S^{T}, \quad \Delta^{i j}=p_{i}\left(1-p_{j}\right), \\
Q^{i}(p(k))=Q_{0}+p_{i} Q_{u}, \quad Y^{m}=Y_{0}+p_{m} Y_{u}, \\
S^{T} B=B R, \quad R K(p)=Y(p), \quad K(p)=R^{-1} Y(p),
\end{gathered}
$$

the constant gains of the desired controller can be obtained as follows:

$$
K_{0}=R^{-1} Y_{0}, \quad K_{u}=R^{-1} Y_{u}
$$

and the closed-system (2.11) is then exponentially mean-square stable for all $p(k) \in\left[\begin{array}{ll}p_{1} & p_{2}\end{array}\right]$.

Proof. Firstly, set

$$
\alpha_{1}(k)=\frac{p_{2}-p(k)}{p_{2}-p_{1}}, \quad \alpha_{2}(k)=\frac{p(k)-p_{1}}{p_{2}-p_{1}}
$$

then, we have

$$
p(k)=\alpha_{1}(k) p_{1}+\alpha_{2}(k) p_{2}
$$

with $\alpha_{i}(k) \geq 0(i=1,2)$ and $\alpha_{1}(k)+\alpha_{2}(k)=1$. Similarly, let

$$
\beta_{1}(k)=\frac{p_{2}-p(k+1)}{p_{2}-p_{1}}, \quad \beta_{2}(k)=\frac{p(k+1)-p_{1}}{p_{2}-p_{1}}
$$

then we have

$$
p(k+1)=\beta_{1}(k) p_{1}+\beta_{2}(k) p_{2}
$$


with $\beta_{i}(k) \geq 0(i=1,2), \beta_{1}(k)+\beta_{2}(k)=1$. From the above transformation, we can easily get

$$
\begin{gathered}
Q(p(k))=\sum_{i=1}^{2} \alpha_{i}(k) Q^{i}, \quad \bar{\Lambda}=\sum_{l=1}^{2} \beta_{l}(k) \bar{\Lambda}^{l}, \\
Y(p(k))=\sum_{m=1}^{2} \alpha_{m}(k) Y^{m}(p) .
\end{gathered}
$$

On the other hand, it is easy to find that

$$
\sum_{i, j, l, m=1}^{2} \alpha_{i}(k) \alpha_{j}(k) \alpha_{m}(k) \beta_{l}(k) \mathbb{M}^{i j l m}<0 .
$$

From (3.22)-(3.27), we can have that (3.5) in Theorem 3.4 is true, then the proof is now complete.

Remark 3.7. The above conclusions can be extended to multiple sensor case of measurement output. In this paper, to make the main idea and the proof more clear and concise, we choose the single sensor.

\section{An Illustrative Example}

In this section, the gain-scheduled static output feedback controller is designed for the discrete-time nonlinear stochastic systems with infinite-distributed delays and missing measurements.

The system parameters are given as follows:

$$
\begin{gathered}
A=\left[\begin{array}{cc}
0.97 & 0 \\
0 & 0.21
\end{array}\right], \quad N=\left[\begin{array}{ll}
0.13 & 0.21 \\
0.28 & 0.33
\end{array}\right], \quad B=\left[\begin{array}{cc}
0.06 & 0 \\
0 & 0.16
\end{array}\right], \\
C=\left[\begin{array}{cc}
0.1 & 0.2 \\
0.15 & 0.23
\end{array}\right], \quad D=\left[\begin{array}{cc}
0.23 & 0 \\
0.15 & 0.18
\end{array}\right], \quad F_{1}=\left[\begin{array}{cc}
0.06 & 0 \\
0 & 0.07
\end{array}\right], \\
F_{2}=\left[\begin{array}{cc}
0.61 & 0 \\
0 & 0.25
\end{array}\right], \quad G=\left[\begin{array}{ll}
0.11 & 0.12 \\
0.18 & 0.12
\end{array}\right], \quad G_{d}=\left[\begin{array}{cc}
0.11 & 0.29 \\
0.18 & 0.09
\end{array}\right], \quad E=\left[\begin{array}{ll}
0.03 & 0.19 \\
0.21 & 0.33
\end{array}\right], \\
p_{1}=0.19, \quad p_{2}=0.51, \quad \sigma^{2}=1, \quad \bar{\mu}=2^{-3} .
\end{gathered}
$$

Set the time-varying Bernoulli distribution sequences as $p(k)=p_{1}+\left(p_{2}-p_{1}\right)|\sin (k)|$ and the sector nonlinear function $f(u)$ is taken as

$$
f(u)=\frac{F_{1}+F_{2}}{2} u+\frac{F_{2}-F_{1}}{2} \sin (u),
$$

which satisfies (2.3). Also, select the initial state as follows: $\rho=[2-2]^{T}$. 


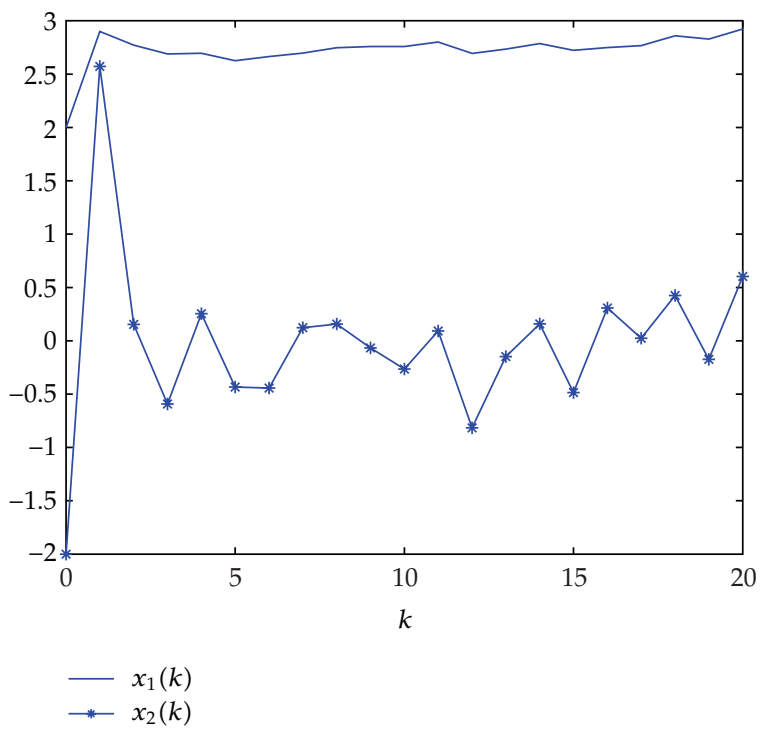

Figure 1: State evolution $x(k)$ of uncontrolled systems.

Table 1: Computing results.

\begin{tabular}{|c|c|c|c|}
\hline$k$ & $p(k)$ & $Q(p(k))$ & $K(p)$ \\
\hline 0 & 0.4593 & {$\left[\begin{array}{cc}3.4334 & -0.2204 \\
-0.2204 & 2.1583\end{array}\right]$} & {$\left[\begin{array}{cc}606.0619 & -529.7133 \\
10.7547 & -16.6786\end{array}\right]$} \\
\hline 1 & 0.4810 & {$\left[\begin{array}{cc}3.4368 & -0.2282 \\
-0.2282 & 2.1833\end{array}\right]$} & {$\left[\begin{array}{ll}607.6342 & -531.0712 \\
10.7201 & -16.6872\end{array}\right]$} \\
\hline 2 & 0.2352 & {$\left[\begin{array}{cc}3.3986 & -0.1400 \\
-0.1400 & 1.9006\end{array}\right]$} & {$\left[\begin{array}{cc}589.8268 & -515.6926 \\
11.1130 & -16.5901\end{array}\right]$} \\
\hline 3 & 0.4322 & {$\left[\begin{array}{cc}3.4292 & -0.2107 \\
-0.2107 & 2.1272\end{array}\right]$} & {$\left[\begin{array}{cc}604.0991 & -528.0183 \\
10.7981 & -16.6679\end{array}\right]$} \\
\hline
\end{tabular}

According to Theorem 3.6, the constant controller parameters $K_{0}, K_{u}$ can be obtained as follows:

$$
K_{0}=\left[\begin{array}{cc}
572.7914 & -500.9808 \\
11.4889 & -16.4972
\end{array}\right], \quad K_{u}=\left[\begin{array}{cc}
72.4419 & -62.5612 \\
-1.5985 & -0.3949
\end{array}\right]
$$

Then, according to the measured time-varying probability parameters $p(k)$, the gainscheduled controller gain $K(p)$ and parameter-dependent Lyapunov matrix can be calculated at every time step $k$ as in Table 1 .

Figure 1 gives the response curves of state $x(k)$ of uncontrolled systems. Figure 2 depicts the simulation results of state $x(k)$ of the controlled systems. The simulation results have illustrated our theoretical analysis. 


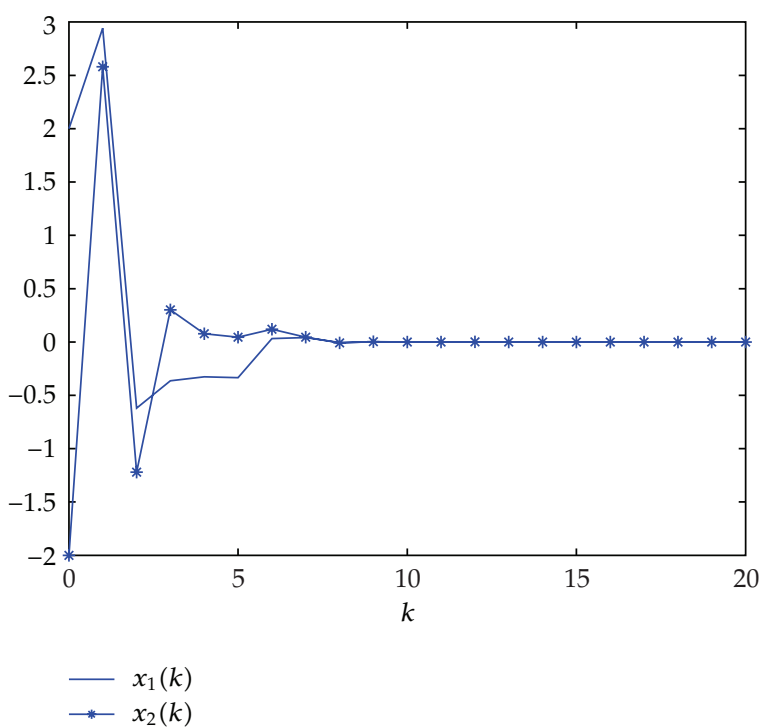

Figure 2: State evolution $x(k)$ of controlled systems.

\section{Conclusions}

In this paper, the problem of gain-scheduled control for a class of discrete stochastic systems with infinite-distributed delays and missing measurements has been studied, the missingmeasurement phenomenon is assumed to occur in a random way, the missing probability is governed by an individual random variable satisfying a certain probabilistic distribution

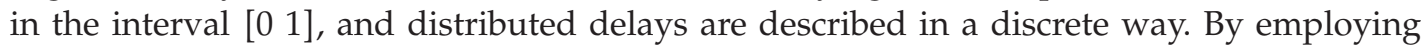
probability-dependent Lyapunov functional, we have designed a gain-scheduled controller with the gain including both constant parameters and time-varying parameters such that, for the admissible missing measurements with time-varying probability, infinite-distributed delays, and noise disturbances, the closed-loop system is exponentially mean-square stable. Moreover, we can extend the main results to more complex and realistic systems, for instance, system with norm-bounded or polytopic uncertainties. Meanwhile, we can also consider dynamic output feedback control problem for discrete stochastic systems with missing measurements by gain-scheduling approach as well as the relevant applications in networked control system or robotic manipulator.

\section{Acknowledgments}

This work was supported in part by the National Natural Science Foundation of China under Grant 61074016, the Program for Professor of Special Appointment (Eastern Scholar) at Shanghai Institutions of Higher Learning, the Program for New Century Excellent Talents in University under Grant NCET-11-1051, the Shanghai Natural Science Foundation of China under Grant 10ZR1421200, the Leverhulme Trust of the UK, the Alexander von Humboldt Foundation of Germany, and the Innovation Fund Project for Graduate Student of Shanghai under Grant JWCXSL1202. 


\section{References}

[1] P. Apkarian and R. J. Adams, "Advanced gain-scheduling techniques for uncertain systems," IEEE Transactions On Control Systems Technology, vol. 6, no. 1, pp. 21-32, 1998.

[2] Y. Y. Cao, Z. Lin, and Y. Shamash, "Set invariance analysis and gain-scheduling control for LPV systems subject to actuator saturation," Systems E Control Letters, vol. 46, no. 2, pp. 137-151, 2002.

[3] W. H. Chen, Z. H. Guan, and X. Lu, "Delay-dependent exponential stability of uncertain stochastic systems with multiple delays: an LMI approach," Systems $\mathcal{E}$ Control Letters, vol. 54, no. 6, pp. 547-555, 2005.

[4] W. J. Rugh and J. S. Shamma, "Research on gain scheduling," Automatica, vol. 36, no. 10, pp. 14011425, 2000.

[5] G. Wei, Z. Wang, and B. Shen, "Probability-dependent gain-scheduled filtering for stochastic systems with missing measure- ments," IEEE Transactions on Circuits and Systems II: Express Briefs, vol. 58, no. 11, pp. 753-757, 2011.

[6] G. Wei, Z. Wang, and B. Shen, "Probability-dependentgain-scheduled control for discrete stochastic delayed systems with randomly occurring nonlinearities," International Journal of Robust and Nonlinear Control, 2012.

[7] Y. Y. Cao, J. Lam, and Y. X. Sun, "Static output feedback stabiliztion: an LMI approach," Automatica, vol. 34, no. 12, pp. 1641-1645, 1998.

[8] J. C. Geromel, C. C. de Souza, and R. E. Skelton, "Static output feedback controllers: stability and convexity," IEEE Transactions on Automatic Control, vol. 43, no. 1, pp. 120-125, 1998.

[9] I. N. Kar, "Design of static output feedback controller for uncertain systems," Automatica, vol. 35, no. 1, pp. 169-175, 1999.

[10] R. E. Benton, Jr. and D. Smith, "Static output feedback stabilization with prescribed degree of stability," IEEE Transactions on Automatic Control, vol. 43, no. 10, pp. 1493-1496, 1998.

[11] E. Prempain and I. Postlethwaite, "Static output feedback stabilisation with $H_{\infty}$ performance for a class of plants," Systems \& Control Letters, vol. 43, no. 3, pp. 159-166, 2001.

[12] V. L. Syrmos, C. T. Abdallah, P. Dorato, and K. Grigoriadis, "Static output feedback-a survey," Automatica, vol. 33, no. 2, pp. 125-137, 1997.

[13] Z. Wang F, Yang, D. W. C. Ho, and X. Liu, "Robust $H_{\infty}$ filtering for stochastic time-delay systems with missing measurements," IEEE Transactions on Signal Processing, vol. 54, no. 7, pp. 2579-2587, 2006.

[14] H. Gao and T. Chen, " $H_{\infty}$ estimation for uncertain systems with limited communication capacity," Institute of Electrical and Electronics Engineers. Transactions on Automatic Control, vol. 52, no. 11, pp. 2070-2084, 2007.

[15] G. Wei, Z. Wang, X. He, and H. Shu, "Filtering for networked stochastic time-delay systems with sector nonlinearity," vol. 56, no. 1, pp. 71-75, 2009.

[16] G. Wei, Z. Wang, and H. Shu, "Robust filtering with stochastic nonlinearities and multiple missing measurements," Automatica, vol. 45, no. 3, pp. 836-841, 2009.

[17] Y. Kuang, H. L. Smith, and R. H. Martin, "Global stability for infinite-delay, dispersive LotkaVolterra systems: weakly interacting populations in nearly identical patches," Journal of Dynamics and Differential Equations, vol. 3, no. 3, pp. 339-360, 1991.

[18] Y. Liu, Z. Wang, and X. Liu, "Robust $H_{\infty}$ control for a class of nonlinear stochastic systems with mixed time delay," International Journal of Robust and Nonlinear Control, vol. 17, no. 16, pp. 1525-1551, 2007.

[19] L. Xie, E. Fridman, and U. Shaked, "Robust $H_{\infty}$ control of distributed delay systems with application to combustion control," Institute of Electrical and Electronics Engineers. Transactions on Automatic Control, vol. 46, no. 12, pp. 1930-1935, 2001.

[20] Z. Wang, G. Wei, and G. Feng, "Reliable $H_{\infty}$ control for discrete-time piecewise linear systems with infinite distributed delays," Automatica, vol. 45, no. 12, pp. 2991-2994, 2009.

[21] Z. Wang, Y. Liu, G. Wei, and X. Liu, "A note on control of a class of discrete-time stochastic systems with distributed delays and nonlinear disturbances," Automatica, vol. 46, no. 3, pp. 543-548, 2010.

[22] G. Wei, G. Feng, and Z. Wang, "Robust $H_{\infty}$ control for discrete-time fuzzy systems with infinitedistributed delays," IEEE Transactions on Fuzzy Systems, vol. 17, no. 1, pp. 224-232, 2009.

[23] S. Boyd, L. El Ghaoui, E. Feron, and V. Balakrishnan, Linear Matrix Inequalities in System and Control Theory, vol. 15, Society for Industrial and Applied Mathematics (SIAM), Philadelphia, Pa, USA, 1994. 
[24] Y. Liu, Z. Wang, J. Liang, and X. Liu, "Synchronization and state estimation for discrete-time complex networks with distributed delays," Transactions on Systems, Man and Cybernetics B, vol. 38, no. 5, pp. 1314-1325, 2008.

[25] F. Yang, Z. Wang, Y. S. Hung, and M. Gani, " $H_{\infty}$ control for networked systems with random communication delays," IEEE Transactions on Automatic Control, vol. 51, no. 3, pp. 511-518, 2006. 


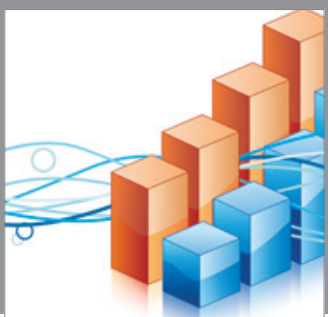

Advances in

Operations Research

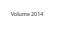

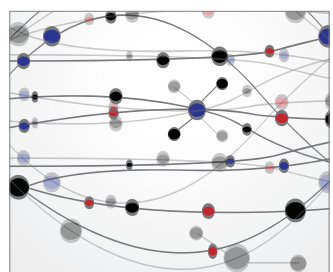

\section{The Scientific} World Journal
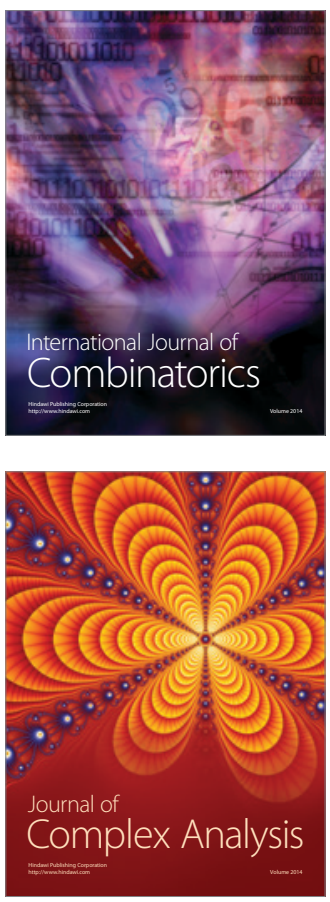

International Journal of

Mathematics and

Mathematical

Sciences
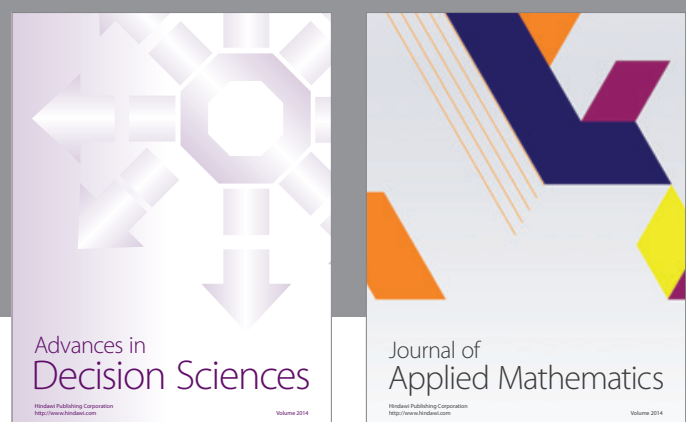

Journal of

Applied Mathematics
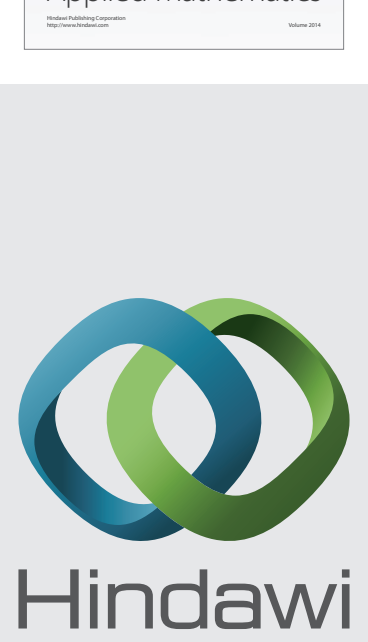

Submit your manuscripts at http://www.hindawi.com
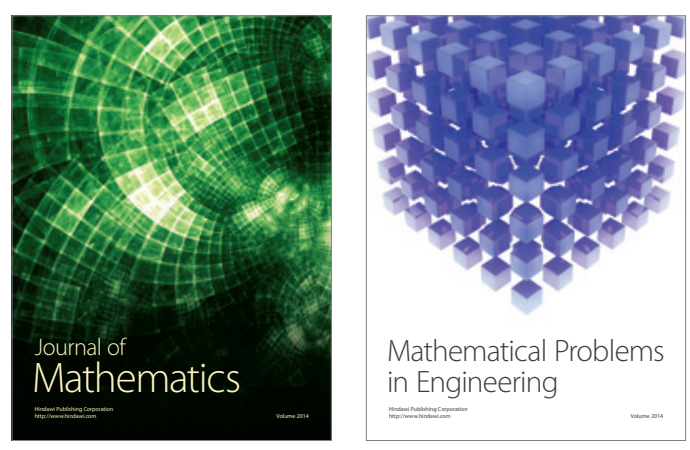

Mathematical Problems in Engineering
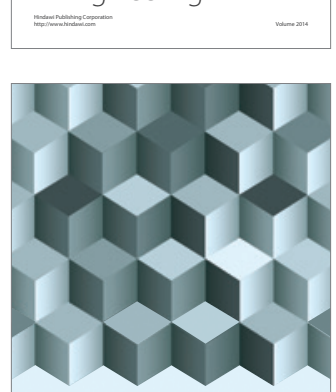

Journal of

Function Spaces
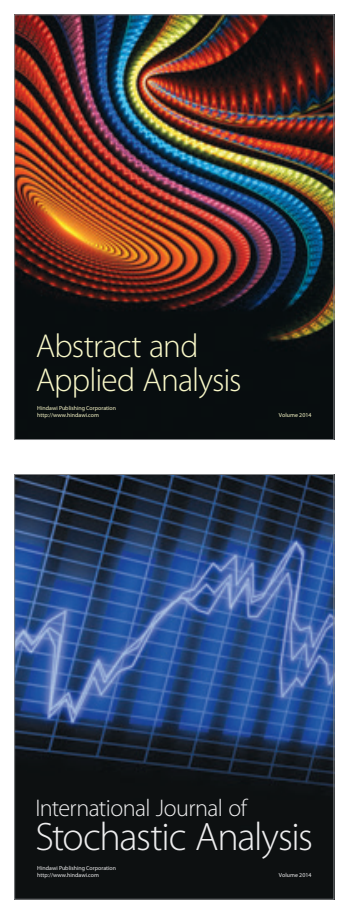

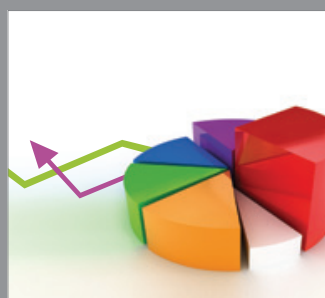

ournal of

Probability and Statistics

Promensencen
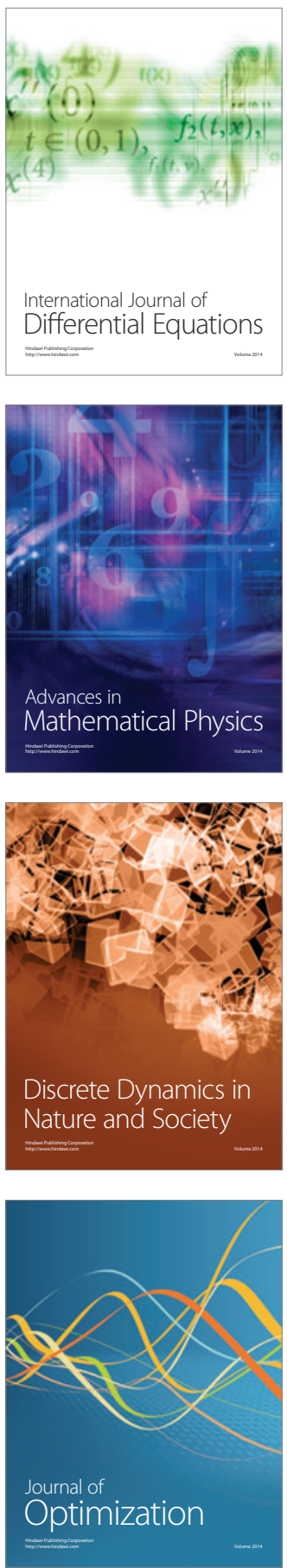